\title{
Influence of counter ion on polyaniline and polypyrrole
}

\author{
D C TRIVEDI \\ Centre for Studies in Conducting Polymers, Central Electrochemical Research Institute, Karaikudi 630006 , India
}

\begin{abstract}
The electronic, magnetic, optical and redox properties of conjugated polymers are greatly influenced by structure, electronegativity, solvation and orientation of a counter ion. The doping by counter ion not only creates a band structure but also stabilizes localized bound states to impart unusual optical and magnetic properties to macromolecular system. When the polymer is in contact with solution of an electrolyte, counter ion tends to be solvated and mobile in the polymer phase, imparting it a property of ion exchange membrane. Here we briefly summarize the influence of anion on properties and electrochemical behaviour of polyaniline and polypyrrole.
\end{abstract}

Keywords. Anion; polyaniline; processibility; intractable; protonation.

\section{Introduction}

The past two decades have witnessed unabated interest in the synthesis and characterization of conjugated polymers that display unusual solid state properties (Trivedi and Srinivasan 1988; Trivedi 1989). The polymers most widely studied up till now are: (i) having a generalized structure, $[-\mathrm{A}-] n$, where $\mathrm{A}$ is an aromatic ring like pyrrole, benzene or thiophene, or (ii) having a generalized structure $[-\mathrm{AB}-] n$ where $\mathrm{A}$ is phenyl and $\mathrm{B}$ is $-\mathrm{NH}-$ imine in polyaniline. These polymers as synthesized are macromolecular cations and hence for the reason of charge compensation they take up counter ion from the electrolyte. Thus the electronic, magnetic and optical properties of polymers become the function of dopant. Due to metallic conductivity these systems are intractable and hence their processibility is a challenge. The conductivity and processibility are twin phenomena, requires the use of concepts which bridge electrochemistry and organic chemistry on the one hand, with the field of condensed matter science on the other. Particularly interesting are systems whose composition can be selectively altered, thereby leading to varying electronic properties for the development of new material having a processibility of a plastic, and electronic conductivity of a metal or a semiconductor.

During the detailed studies on use of variety of dopants, we observed that conductivity and processibility of polyanilines not only depends on protonation level but also is strongly influenced by counter anion. Similarly it has been found that pyrrole on polymerization yields a powdery material in aqueous sulphuric acid medium (Dall'Olio et al 1968), whereas in presence of $\mathrm{BF}_{4}^{-}, \mathrm{ClO}_{4}^{-}$ and $\mathrm{PF}_{6}^{-}$under nonaqueous condition gives a free standing film (Diaz et al 1979). Recently it has been shown that introducing large size dopants (Zhong et al 1990), can alter ion exchange property of a polypyrrole (PPy) film. Thus electrical conductivity, thermal stability, processibility, and electrochemical growth not only depends on oxidation state of a polymer but are also dependent on the nature of counter anion which can be defined in terms of its electronegativity, molecular size, orientation and solvation (Trivedi 1997; Zotti et al 1988).

Polyaniline has been known almost for 136 years as aniline black (Letheby 1862). However, its systematic synthesis and structure are of recent origin (MacDiarmid et al 1985). Polyaniline (PA) though environmentally stable is intractable. Polyaniline is built from reduced $(-\mathrm{B}-\mathrm{NH}-\mathrm{B}-\mathrm{NH})$ and oxidized $(-\mathrm{B}-\mathrm{N}=\mathrm{Q}=\mathrm{N}-)$ repeating units, where $B$ denotes a benzenoid and $Q$ denotes a quinoid ring. Thus the different ratios of amine to imine yield various structures, such as leucoemeraldine (a reduced form), emeraldine base (neutral undoped form), emeraldine salt ( $50 \%$ oxidized, doped form), and perinigraniline (fully oxidized form). The various forms are shown in figure 1 . The conductivity of polyaniline depends not only on oxidation state but also on the degree of anions, to maintain charge neutrality. This suggests that the behaviour of polyaniline depends on the $\mathrm{pH}$ as well as on the counter ion of the Bronsted acid used for doping. The protonation equilibria involves exclusively the quinone diimine segment of the polymer chain, having two imine nitrogens with $\mathrm{pK}_{\mathrm{a} 1}=1.05$ and $\mathrm{pK}_{\mathrm{a} 2}=2.5$ (Haung et al 1987). The protons are added to $-\mathrm{N}=$ sites while the number of electrons in the chain remain constant (Schacklette et al 1988). However, the functionality present in the dopant determines its processibility. Cao et al (1992) reported that when camphor 10 sulphonic acid is used as dopant, the resulting polymer is soluble in solvents like chloroform. Therefore, it is reasonable to expect that electrochemical and other properties of the conductive polymer will 
be influenced by the nature of the incorporated counter ion.

Polypyrrole (PPy) is known since 1916 (Angeli 1916), however, its semiconducting properties were first investigated by McNeill et al (1963). PPy was first electrochemically synthesized as a black powdery deposit in aqueous sulphuric acid medium anodically by Dall'Olio et al (1968). In 1979 (Diaz et al 1979) reported electrochemical synthesis of an electrically conducting free standing film of good mechanical strength. The electrochemical method facilitated incorporation of variety of counterman into polymer film. Since the oxidation potential of PPy is lower than that of monomer, thus the polymer is simultaneously oxidized during polymerization and hence counter ions from electrolyte are incorporated into growing polymer in order to maintain electrical neutrality.

Polypyrrole (PPy) can be viewed as a carbon chain with structure of polyacetylene stabilized by the heteroatom and has a nondegenerate ground state related to nonenergetic equivalence of two limiting mesomeric

(a)<smiles>CNc1ccc(NC2=CC=C(Nc3ccc(Nc4ccc(I)cc4)cc3)CC2)cc1</smiles>

(b)<smiles>CNc1ccc(Nc2ccc(N=C3C=CC(=Nc4ccc(C)cc4)C=C3)cc2)cc1</smiles>

(c)

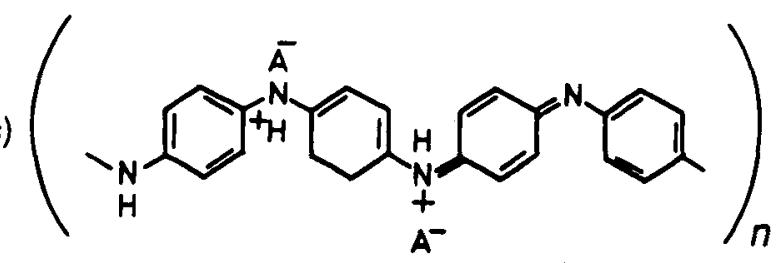

(d)<smiles>CN=C1C=CC(=Nc2ccc(N=C3C=CC(=Nc4ccc(C)cc4)C=C3)cc2)C=C1</smiles>

Figure 1. Various forms of polyaniline: (a) leucoemeraldine; (b) emeraldine base; (c) emeraldine salt; (d) pernigraniline. forms, aromatic and quinoid. In polymerization of pyrrole, the first step consists of the oxidation of the monomer to its radical cation. The second step involves the coupling of two radical cations to yield a dihydrodimer dication that leads to a dimer after loosing two protons and rearomatization. The dimer has less oxidation potential than monomer, undergoes further coupling with a monomeric radical, subsequently electrochemical and chemical steps occur until oligomer becomes insoluble in the electrolyte and precipitates on to the electrode surface. In pyrrole polymerization presence of water is essential to eliminate protonation of pyrrole and therefore, the conductivity of PPy decreases as the anion basicity increases. The polymerization process requires 2-2-2.4 Faraday/mole. 2 Faraday are required for the oxidation of monomer and the remaining are required to generate the macro cation. Thus this corresponds to reversible oxidation (doping) of the polymer.

The doping-dedoping is a complex phenomena governed by many factors, such as ion pair formation which can occur within the doped polymer between the dopant and positive charge on the polymer chain, resulting in the diffusion of cation from electrolyte to keep polymer chain electrically neutral. These cationic species owing to their smaller ionic size have greater mobility, therefore continuous doping-dedoping by cycling the polymer electrode between extreme potential limits yields a polymer free from ion pair as well as from oligomeric impurities. Therefore, it is inevitable that kinetics of doping-dedoping and polymerization is influenced by steric effects associated with counter ion like:

- adsorption on working electrode;

- redox potential;

- the ionic charge;

- ionic size;

- solvation shell;

- ionization constant of the electrolyte;

- concentration of electrolyte;

- electrochemical potential of doping;

- diffusion coefficient of a dopant, which varies with solvent, concentration of electrolyte, temperature and applied potential.

These aspects are important because polymer undergoes substantial volume changes during doping and dedoping processes. These dimensional changes influence mechanical strength of a polymer and have been utilized to convert electrical energy to mechanical energy (Baughman 1996).

Not all dopants can induce charge transport in polymer. The charge transport depends upon the redox energy of the host and guest molecules because transfer of an electron is from high to low redox energy. Initially the charge transfer on doping is between the dopant molecule and the proximal polymer site, but subsequently some diffusion of polymer charge to the dopant site can be 
expected, generating thereby pair states in polymer. In equilibrium, the number of polymer pair states are equal to the number of ionized dopants. ESR (Javadi et al 1988), electron energy loss spectroscopy (Litzemann et al 1989), NMR (Raghunathan et al 1996) and thermopower studies (Raghunathan et al 1993) support these findings. These observations have given rise to speculation that ferromagnetism can be induced in these conjugated systems.

\section{Experimental}

The method of synthesis of emeraldine salt by chemical oxidative polymerization and electrochemical polymerization has been discussed in detail elsewhere (Trivedi 1997). In the present study, we used the following protonic acids as dopants for polyaniline: 2,5 dimethylbenzene sulphonic acid, 1,2-dimethylbenzene sulphonic acid, 3-chloro-4-hydroxy benzenesulphonic acid, 2methyl-4-hydroxy benzenesulphonic acid, 4-hydroxy-mbenzene disulphonic acid, 2,4-dihydroxy- $m$-benzenedisulphonic acid, 2,4-hydroxybenzene sulphonic acid and oxalic acid.

The electrochemical polymerization and characterization of polypyrrole were carried out in sodium salt of dodecyl benzenesulphonic acid, lithium perchlorate and tetralkylammonium salts. Aromatic sulphonic acid used in these studies was synthesized and purified as per standard synthetic organic chemistry methodologies.

$\mathrm{X}$-ray diffractograms for all polymers were recorded using $\mathrm{CuK}_{\alpha}$ radiation $(\alpha=1.5418 \mathrm{~A})$. The diffused reflectance UV-visible spectra for thin films were recorded on Hitachi-U-3400 spectrophotometer and FTIR in $\mathrm{KBr}$ medium on Perkin Elmer FTIR spectrometer. Thermal studies were carried out under nitrogen at flow rate of $100 \mathrm{ml} / \mathrm{min}$ with heating rate of $20 \mathrm{C} / \mathrm{min}$ on Perkin Elmer 7 thermal analyser.

The cyclic voltammetric studies were carried out using Tacussel BI-PAD Bipotentiostat coupled with an X-Y recorder (BBL model SE 780) and PARC universal programmer. The $0.25 \mathrm{~cm}^{2}$ platinum electrodes were used. All electrode potential values reported in this paper are referenced to saturated calomel electrode (SCE).

All chemicals used were analytical grade. All solutions were thoroughly purged with Ar. The platinum electrodes were polished with finer grade alumina $(0.05 \mu \mathrm{m})$ before use.

\section{Results and discussion}

\subsection{Electrochemical studies}

3.1a Polyaniline: The cyclic voltammetric studies during growth of a polymer film suggests that on passing same amount of current under identical conditions thicker polymer film formation occurs in the presence of oxidizing ions like $\mathrm{NO}_{3}^{-}$and $\mathrm{ClO}_{4}^{-}$(Duic et al 1994). However, the anion which promotes the growth of polymer are also responsible for degradation of polymer film by resulting in over oxidation. The general mechanism for oxidative polymerization of aniline at $\mathrm{pH} 1$ and its simultaneous degradation are illustrated in equation (1). Thus, according to this equation two parallel sets of reaction occur: polymer growth and its degradation. As the polymer builds up, delocalization also increases which inhibits the autocatalytic growth of the polymer leading thereby to oxidative degradation of the dimer to quinone.

\subsection{Cyclic voltammetric studies}

The growth pattern of polyaniline in benzenesulphonic acid is given in figure 2 , where a middle peak indicates the degradation to quinone during synthesis. This position of middle peak due to quinone was confirmed by adding quinone during synthesis. The growth of the polyaniline film on electrode substrate was performed by a cyclic potential sweep method by switching the potential between -0.2 and $+0.8 \mathrm{~V}$ vs SCE for aromatic sulphonic acid and -0.2 to $1.0 \mathrm{~V}$ vs SCE for oxalic acid medium by passing charge of $0.32 \mathrm{C} / \mathrm{cm}^{2}$. The cyclic voltammetric (CV) response of the polyaniline film was recorded in an electrolytic medium (without the monomer); the peak potential values are given in table 1.

The cyclic voltammogram (figure 3 ) gave three peaks in all cases except oxalic acid. The first oxidation peak is assigned to the transfer of a single electron giving rise to a radical cation, and the third peak to the transfer of another electron leading to the formation of diradical
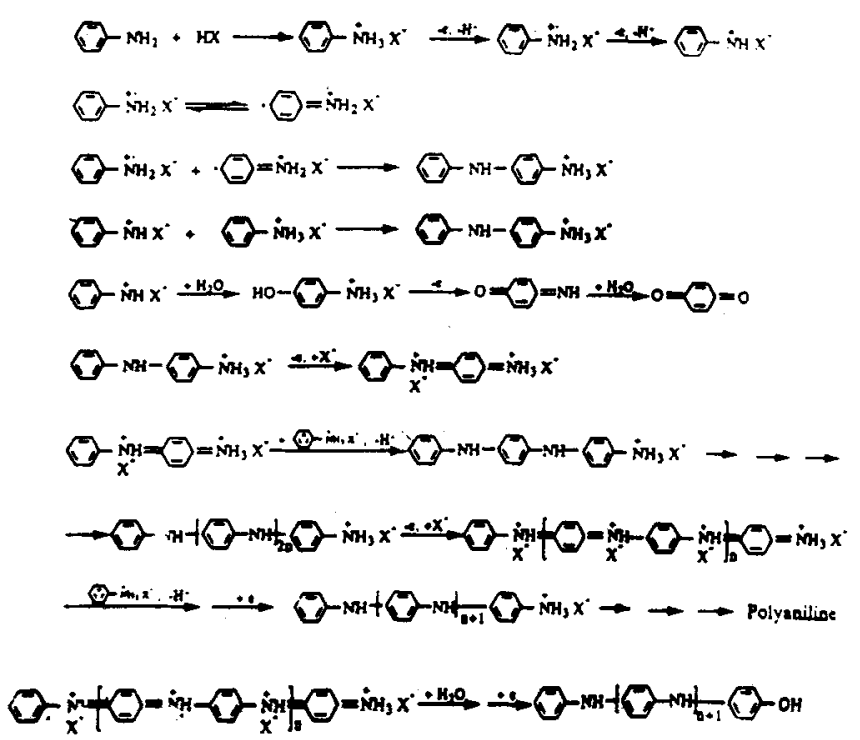

Equation (1). Polymerization mechanism of aniline to polyaniline. 
Table 1. Electrochemical data on polyaniline.

Anodic

Aromatic sulphonic acid

$\begin{array}{lccc}\text { I peak } & \text { II peak } & \text { III peak } & \text { Reference } \\ 0.170 & - & 0.620 & \text { This work } \\ 0.580 & - & 0.860 & \text { This work } \\ 0.200 & - & 0.500 & \text { This work } \\ 0.170 & - & 0.500 & \text { This work } \\ 0.180 & - & 0.580 & \text { This work } \\ 0.220 & - & 0.670 & \text { This work } \\ 0.180 & - & 0.540 & \text { This work } \\ 0.140 & 0.460 & 0.720 & \text { Trivedi and Dhawan (1993a, c) } \\ 0.140 & - & 0.720 & \text { Trivedi and Dhawan (1993a, b) } \\ 0.100 & 0.410 & 0.710 & \text { Dhawan and Trivedi (1993a) } \\ 0.130 & 0.400 & 0.700 & \text { Dhawan and Trivedi (1991) } \\ 0.125 & 0.400 & 0.670 & \text { Dhawan and Trivedi (1992) } \\ 0.090 & 0.360 & 0.625 & \text { Dhawan and Trivedi (1992) } \\ 0.240 & - & 0.460 & \end{array}$

dication, followed by loss of two protons as given in equation (2). The middle peak is assigned to the hydrolysis product of the polymer like benzoquinone or hydroquinone as shown in equation (1); which gets entrapped in

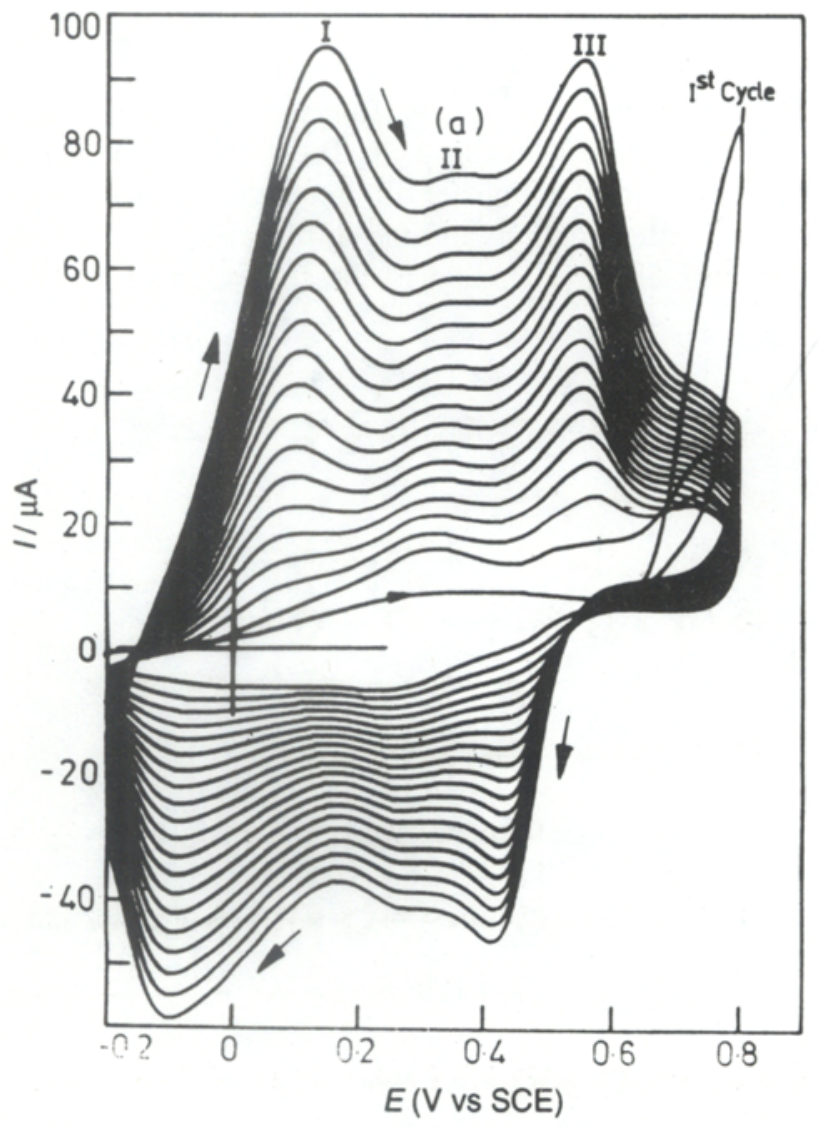

Figure 2. Growth pattern of polyaniline in $1 \mathrm{M}$ benzene sulphonic acid (aniline, $0.1 \mathrm{M}$; sweep rate, $50 \mathrm{mV} / \mathrm{sec}$ ). polymer matrix during synthesis. However, such degradation is ruled out in presence of reducing anions like oxalate. It has been observed that polyaniline film grown in a reducing medium like oxalic acid has a very high stability. In this system the surface electron transfer occurs at $240 \mathrm{mV}$ and protonation around $460 \mathrm{mV}$ vs $\mathrm{SCE}$. This narrow potential range observed in oxalic acid medium is particularly suitable for electrochromic display due to reduction in time required for cycling the film between extreme potentials. The growth of polyaniline in $1 \mathrm{M}$ oxalic acid is given in figure 4 to show how the degradation of polyaniline can be minimized.

3.2a Membrane properties of polyaniline: The membrane properties of polyaniline were investigated on electrodeposited polyaniline on platinum electrode of $1 \mathrm{~cm}^{2}$. We found that the slope in the curve of potential vs $\log (C)$ concentration were less than $59 \mathrm{mV}$ indicating that in addition to anion transport cation transport also<smiles>CNc1ccc(NC)cc1</smiles>
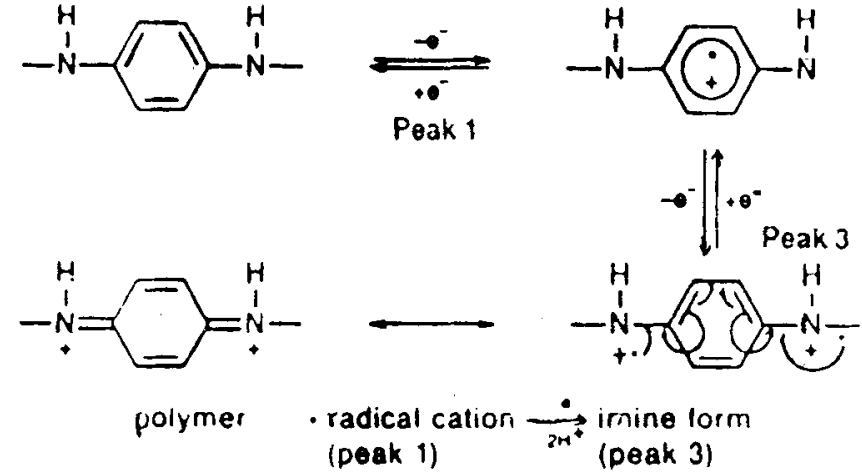

Equation (2). Explains the $\mathrm{CV}$ peaks of polyaniline. 
occurs. Slopes of $45-50 \mathrm{mV}$ were observed for $\mathrm{F}^{-} \mathrm{Cl}^{-}$, $\mathrm{Br}^{-}, \mathrm{NO}_{3}^{-}$, and $\mathrm{ClO}_{4}^{-}$, whereas slope of $15 \mathrm{mV}$ was observed in aromatic sulphonate medium indicating that anion size influences transport across the membrane.

At $\mathrm{pH} 1$ in aromatic sulphonic acid medium negative slopes were observed indicating that anion permeability decreases and $\mathrm{H}^{+}$are exchanged.

3.2b Electronic spectra: The diffused reflectance spectra on platinum substrate gave well-resolved peaks at 320 $330 \mathrm{~nm}, \quad 420-430 \mathrm{~nm}$ and $790-900 \mathrm{~nm}$. These peak positions were found to be dependent on doping level. At $25 \%$ doping level absorption peaks were observed at 317,410 and $670 \mathrm{~nm}$ and at 50\% doping level (maximum achievable doping level) peaks were observed at 320 , 420 and $840 \mathrm{~nm}$. The $\left(\pi-\pi^{*}\right)$ transition was observed at $427 \mathrm{~nm}$ due to semiquinone structure and band due to trapped excitons was found around $648 \mathrm{~nm}$ in polyaniline-oxalic acid system, however, in all other systems it was observed at $850 \mathrm{~nm}$. The polyaniline film grown in oxalic acid, on dedoping and redoping in sulphuric acid medium, the $648 \mathrm{~nm}$ band shifts to $850 \mathrm{~nm}$ due to bipolarons, suggesting that absorption band at $648 \mathrm{~nm}$

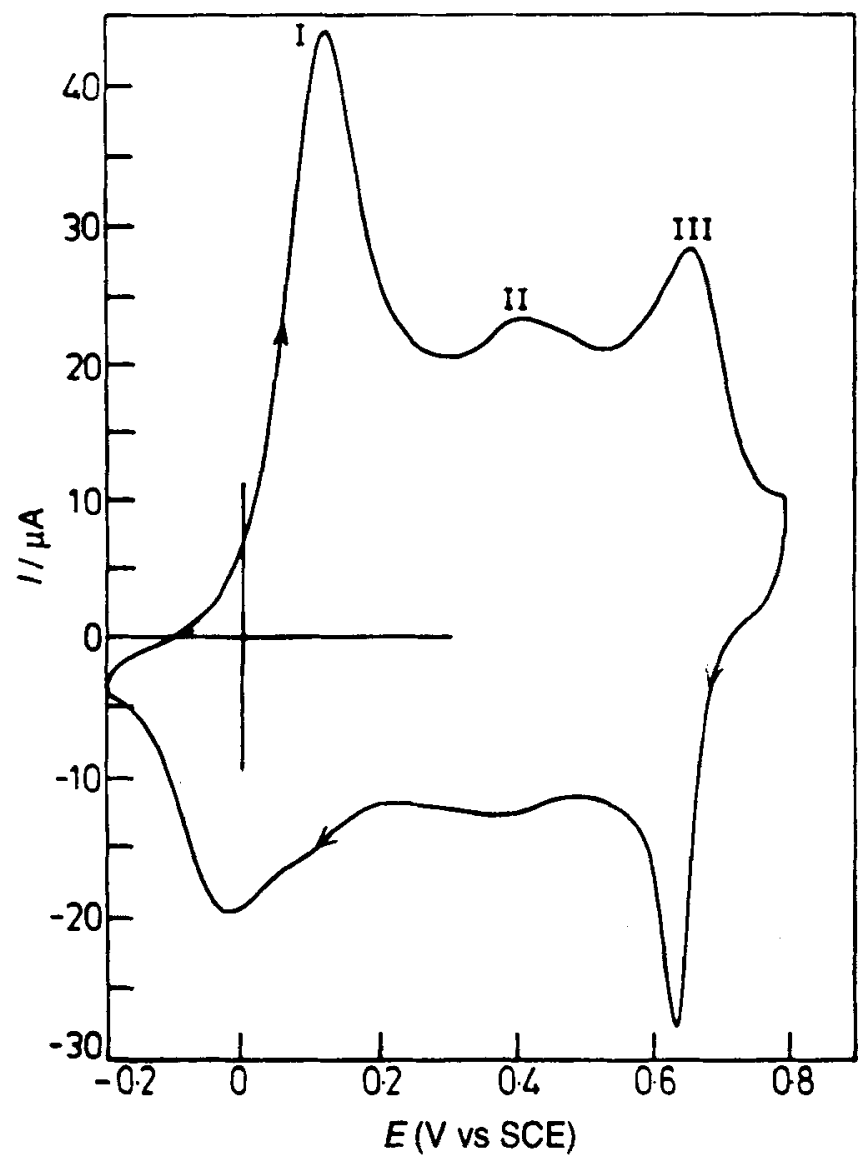

Figure 3. Cyclic voltammogram of polyaniline in $1 \mathrm{M}$ benzene sulphonic acid (Sweep rate, $50 \mathrm{mV} / \mathrm{sec}$ ). in PAn-oxalic acid system is not due to lower conjugation in polymer but is essentially due to polarons.

3.2c 'HNMR studies: The 'H NMR of doped (with various dopant) polyaniline in DMSOd $_{6}$ gave broad resonance aromatic peaks around 7.6 and $7.18 \mathrm{ppm}$ due to aromatic protons of the aniline ring system. The $\mathrm{NH}$ proton signals were observed between $3.8 \mathrm{ppm}$ and $3.6 \mathrm{ppm}$. In all the cases undoped samples gave aromatic proton signals at $7 \mathrm{ppm}$ and $\mathrm{NH}$ proton signal at $3.34 \mathrm{ppm}$.

3.2d FTIR studies: The main FTIR characteristic bands observed in $\mathrm{KBr}$ medium are:

$\begin{array}{ll}\text { Nitrogen-benzenoid-quinoid } & -1572-1560 \mathrm{~cm}^{-1} \\ \text { N-H stretching } & -1490-1495 \mathrm{~cm}^{-1} \\ \text { C-C stretching } & -1300-1303 \mathrm{~cm}^{-1} \\ \text { C-N stretching } & -1250-1260 \mathrm{~cm}^{-1} \\ \text { SO2 } & -1170-1160 \mathrm{~cm}^{-1} \\ \text { C-H band } & -1130-1140 \mathrm{~cm}^{-1} \\ \text { SO3- } & -1020-1040 \mathrm{~cm}^{-1} \\ \text { C-H out of plane } & -798-802 \mathrm{~cm}^{-1}\end{array}$

The $\mathrm{C}-\mathrm{H}$ out of plane band $798-802 \mathrm{~cm}^{-1}$ indicates the para coupling in the polymer.

3.2e Electron spin resonance: In all cases, room temperature ESR-line exhibits pronounced asymmetry with $\Lambda / \mathrm{B}$ ratio at about 1.4 . This ratio decreases to 1.2 at $77 \mathrm{~K}$. The electrochemically synthesized PPy film gave $g=2.0024$, and $\triangle H p p \quad 0.969 \mathrm{G}$. Polyaniline gave $g=2.0028$ and $\Delta H p p 4.95 \mathrm{G}$. In case of polypyrrole, line shape remained unchanged from room temperature to liquid nitrogen temperature indicating strong paramagnetism (detailed study would be reported elsewhere).

3.2f $X$-ray diffraction: In all cases broadening of peaks occurs indicating amorphous nature of materials. The four sets of $2 \theta$ values were observed ranging from 18 to 26 .

$3.2 \mathrm{~g}$ Thermal stability: In all cases first weight loss (3\%) occurs between $60-100^{\circ} \mathrm{C}$ due to moisture. However, the stability of a doped state becomes the function of a dopant. The dopant loss was found to be around $40 \%$ by weight. The data are recorded in table 2 .

The anion effect is possibly related to the hydration shell of anion as suggested by Desilvestro and Scheifele (1993) who have shown that the hydration enthalpies and Gibbs free energies show a good correlation with polymerization rates. However, onset potential for oxidation (polymerization) of aniline is independent of anion and its self-catalyzing reaction and obeys the law $1 / n F A=K c$, where $K c$ is the autocatalytic rate constant 
and has a value of approximately $0.47 \mathrm{~s}^{-1}$ for a film of $140 \mathrm{~nm}$ (Shim and Park 1989).

The use of aromatic functionalized sulphonic acid as a dopant induces certain changes in properties of poly-

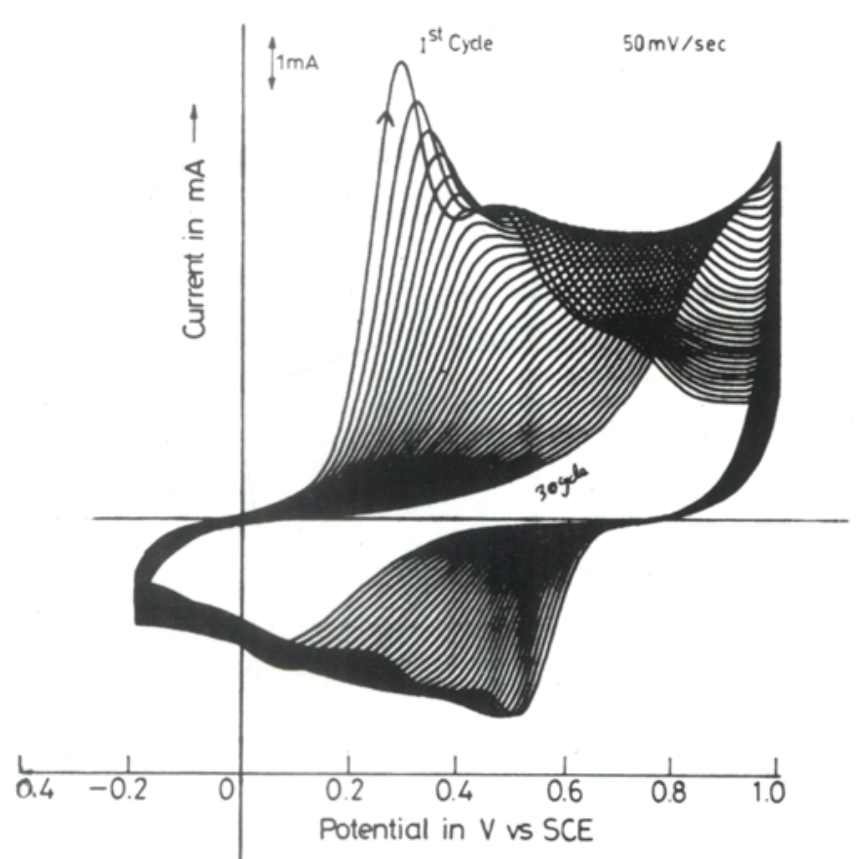

Growth of polyaniline 1M Oxalic acid

Figure 4. Growth of polyaniline in $1 \mathrm{M}$ oxalic acid (aniline $0.1 \mathrm{M}$; sweep rate, $50 \mathrm{mV} / \mathrm{sec}$ ).

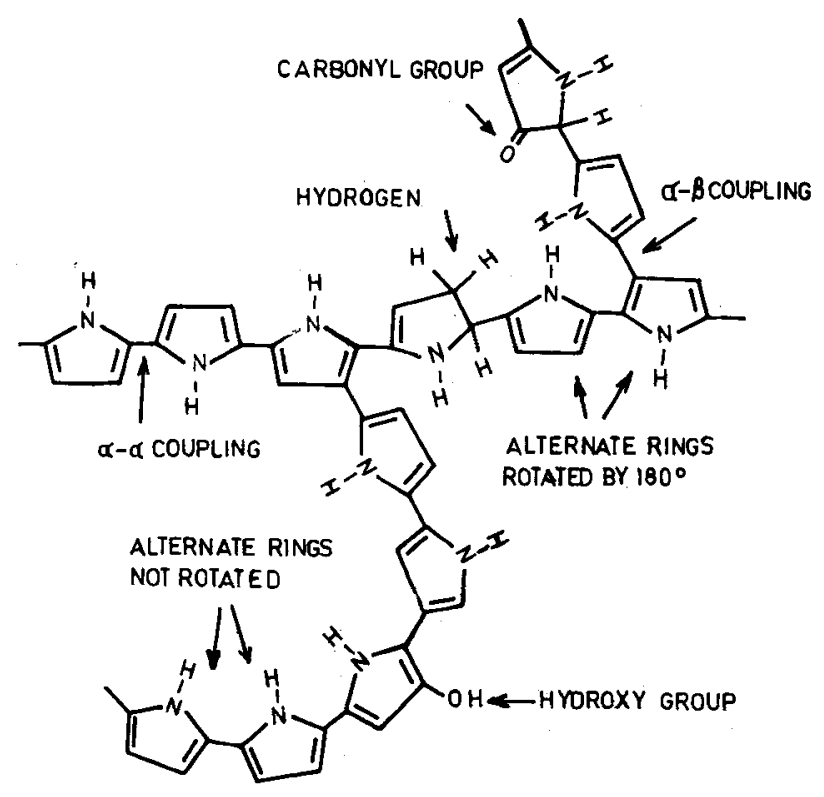

Figure 5. Various defects in the polypyrrole (PPy) structure in presence of oxidizing anions. aniline via formation of ternary system involving polymer, dopant and solvent. Solvation of polyaniline is a result of a number of interactions between polymer dopant and solvent such as Borne-type, hydrogen bonding, hard-soft acid base interactions and Lewis acid base (donoracceptor interactions). The change in structure of polymer cannot be ruled out due to restructuring of the solvent in presence of ions. Thus functionalized protonic acid yields a DMSO, chloroform, xylene and various common organic solvent soluble conducting polyaniline, from which free standing film can be easily obtained.

Currently, research in polyaniline is centred around finding a suitable dopant that can induce formation of blend with commercially viable polymers such as polyolefins, polyesters, poly(methyl methacrylate). In these systems, counter ion acts as a surfactant, for example dodecyl benzene sulphonic acid and camphor sulphonic acid can yield a polyblend with polymethyl methacrylate with $2 \%$ polyaniline with electrical conductivity of $100 \mathrm{~S} \mathrm{~cm}^{-1}$ (Heeger 1995). Studies have indicated that using suitable dopant polyaniline can find applications in control of EMI shielding (Trivedi and Dhawan 1993a, b, c), batteries (Kumar et al 1996), corrosion inhibitors (Sathyanarayan et al 1992, 1994; Dhawan and Trivedi 1993a, b 1995) and as sensors (Dhawan et al 1997).

\subsection{Polypyrrole [PPy]}

Polypyrrole is one of the polymers that has attracted equal attention from physicists and chemists, because of its simplicity of structure and low oxidation potential which favours the formation of 2,5-bonded polymer as a free-standing film on an anode surface. The poly merization of pyrrole can be effected in presence of host of electrolytes under aqueous or nonaqueous conditions. Due to low oxidative polymerization potential, pyrrole can be polymerized by vapour phase oxidation in presence of suitable mild oxidant, e.g. ferric ion (Dhawan and Trivedi 1993a, b). Due to low oxidation potential of polypyrrole than polymerization potential of pyrrole, the oxidizing anions like $\mathrm{NO}_{3}^{-}$and $\mathrm{ClO}_{4}^{-}$in presence of protonic impurities can yield a polymer having other than 2,5 linkage having a carbonyl and hydroxyl group (see figure 5). Thus conjugational length depends on the syntheses conditions, like temperature, concentration of monomer and electrolyte and electrochemical potential. At lower electrochemical potential conjugational defects are reduced because of slower rate of reaction. Lowering the temperature during synthesis can further lower the rate of reaction. The polymerization mechanism is given in equation (3).

A unique aspect of the electrochemical behaviour of the polypyrrole film is that it is inherently redox electroactive. The broad peak and its shape in cyclic voltammogram (figures 6 and 7) suggest that redox reaction 
Table 2. Thermal stability of electrochemically synthesized polyaniline.

\begin{tabular}{lccc}
\hline Dopant & $\begin{array}{c}\text { Moisture expulsion } \\
\text { temperature }\left({ }^{\circ} \mathrm{C}\right)\end{array}$ & $\begin{array}{c}\text { Dopant expulsion } \\
\text { temperature }\left({ }^{\circ} \mathrm{C}\right)\end{array}$ & $\begin{array}{c}\text { Decomposition } \\
\text { temperature }\left({ }^{\circ} \mathrm{C}\right)\end{array}$ \\
\hline 5-Sulphosalicylic acid & 80 & 238 & 472 \\
2,4-Dihydroxy-m-benzene disulphonic acid & 71 & 369 & 472 \\
2-Methyl-4-hydroxy benzenesulphonic acid & 100 & 468 & 549 \\
2-Hydroxy benzene sulphonic acid & 60 & 329 & 638 \\
2,5-Dimethyl benzene sulphonic acid & 64 & 455 & 654 \\
4-Hydroxy benzene 3,6 disulphonic acid & 90 & 356 & 700 \\
\hline
\end{tabular}

Table 3. Electrochemical data on polypyrrole.

\begin{tabular}{lccc}
\hline Electrolyte & $E_{\mathrm{pa}}(\mathrm{mV})$ & $E_{\mathrm{pc}}(\mathrm{mV})$ & Conductivity $(\mathrm{s} / \mathrm{cm})$ \\
\hline In Acetonitrile & & & \\
& & & \\
$\mathrm{LiClO}_{4}$ & -110 & -440 & 100 \\
$\mathrm{Me}_{4} \mathrm{NPF}_{6}$ & -160 & -325 & 50 \\
$\mathrm{Et}_{4} \mathrm{NBF}_{4}$ & -100 & -300 & 20 \\
$\mathrm{Et}_{4} \mathrm{NClO}_{4}$ & -090 & -290 & 100 \\
$\mathrm{Bu}_{4} \mathrm{NBF}_{4}$ & -110 & -260 & 20 \\
In aq. medium & & & \\
Dodecylbenzene sulphonate & +170 & & 01 \\
\hline
\end{tabular}

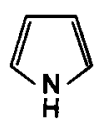

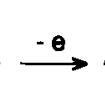

(a)<smiles>CC(C)C1CCCC1</smiles>

(e)<smiles>c1c[nH]c([C@H]2CCCN2)c1</smiles>

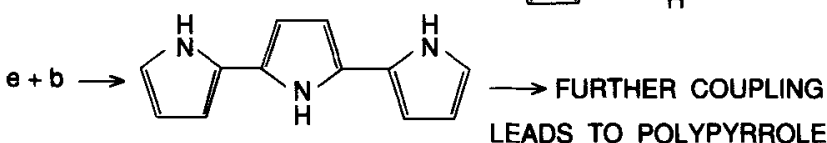

LEADS TO POLYPYRROLE<smiles>[Y]c1cc(-c2ccc(-c3ccc(-c4ccc(-c5ccc(C)[nH]5)[nH]4)[nH]3)[nH]2)[nH]c1C(C)O[Na]</smiles>

Equation (3). Polymerization mechanism of pyrrole to polypyrrole.
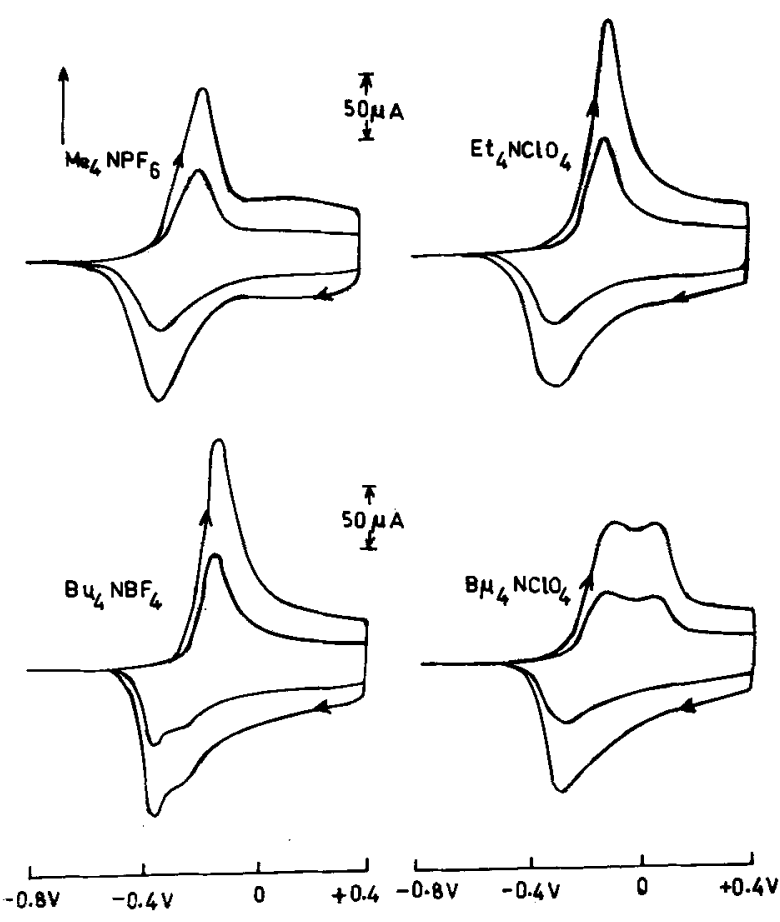

CYCLIC VOLTAMMOGRAMS (Pt)POLYPYRROL:(20nMTHICK) IN $\mathrm{CH}_{3} \mathrm{CN}$ SWEEP RATES:50 AND $100 \mathrm{mV}^{\mathrm{e}^{-1}}$

Figure 6. Cyclic voltammogram of polypyrrole at $50 \mathrm{mV} / \mathrm{sec}$ and $100 \mathrm{mV} / \mathrm{sec}$ in $\left(\mathrm{CH}_{3}\right)_{4} \mathrm{NPF}_{6},\left(\mathrm{C}_{2} \mathrm{H}_{5}\right)_{4} \mathrm{NClO}_{4},\left(\mathrm{C}_{4} \mathrm{H}_{9}\right)_{4} \mathrm{NBF}_{4}$, $\left(\mathrm{C}_{4} \mathrm{H}_{9}\right)_{4} \mathrm{NClO}_{4}$. 
of polypyrrole is not strictly Nerstian. This behaviour indicates slow ion mobility in the polymer film. Using tetraalkyl ammonium salt (fluoroborate and perchlorate), we found that $10 \%$ excess of charge gets consumed, indicating that four pyrrole units consumed one electron to give a macro cation.

It is not clear to what extent the broadening of peak is due to repulsive interactions between the electroactive sites and/or due to the electrochemical non-equivalence of the sites. In all cases peak width are more than $90 \mathrm{mV}$, which is not expected for surface electron transfer reaction.

As can be seen from cyclic voltammograms, using various tetraalkyl salts, that the electrolyte $\mathrm{Bu}_{4} \mathrm{NBF}_{4}$ influences reduction reaction, while $\mathrm{Bu}_{4} \mathrm{NClO}_{4}$ influences oxidation reaction (figure 6 ). When we replaced tetralkyl salt by sodium dodecyl benzene sulphonate using aqueous medium, the peak observed were very broad, and cathodic peak at higher sweep rate tended to bifurcate indicating the retention of the large surfactant anion in the polymer matrix during reduction process (figure 7). This behaviour indicates that fixed anionic charges can be incorporated on polypyrrole film to be utilized as the ion exchange membrane.

Table 3 summarizes some of the electrochemical parameters on polypyrrole in presence of various dopants.

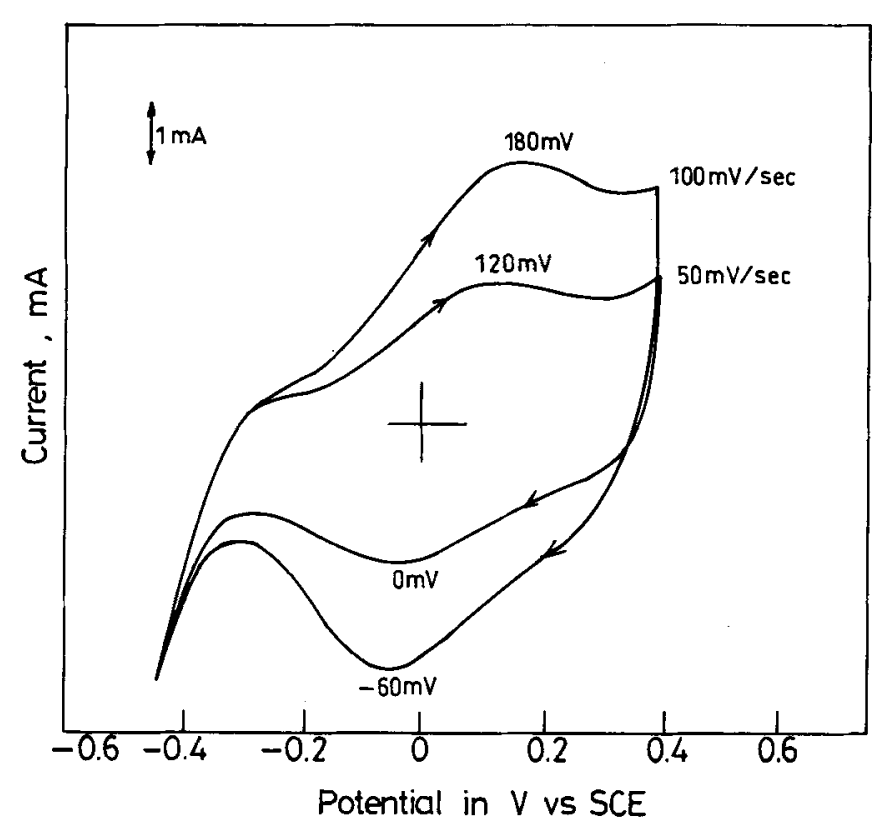

Cyclic voltammogram $(P t)$ poly pyrrole $(20 \mathrm{~nm}$ thick) in $0.1 \mathrm{M}$ Sodium dodecyl benzene sulphonate.

Figure 7. Cyclic voltammogram of polypyrrole at $50 \mathrm{mV} / \mathrm{sec}$ and $100 \mathrm{mV} / \mathrm{sec}$ in $1 \mathrm{M}$ aqueous solution of dodecyl benzene sulphonate. 3.3a Electrochemical growth: The growth of conducting polymer film has been a subject of intensive studies by various techniques to understand its various properties like ion transport and optical. In principle, the growth of the conducting polymer can be compared with electrodeposition of metals, the difference being that the former is an anodic reaction. The filming of an anode surface during electrolysis of a monomer in a suitable electrolytic medium occurs via oxidative generation of radical cations near the vicinity of an anode which on further coupling leads to precipitation of conducting polymer leading to filming of an anode surface suggesting that particles are added one at a time to a cluster or aggregate of particles via random walk trajectories that is to say that competing growth of polymer from anode surface leads to formation of a cluster. This formation of a cluster can be considered as an alloy of macromolecule with counter ion giving it a distinct entity.

The electrodeposition reaction from solution phase is a transformation reaction, thus there is a tendency in the system between order and disorder, which results in thermodynamic fluctuations leading to formation of disordered phase. For example Kim et al (1991), using ellipsometry as a tool, have reported that during polypyrrole deposition from $0.2 \mathrm{M}$ aq. potassium nitrate solution, at first the film grows linearly up to $15 \mathrm{~nm}$ in $50 \mathrm{~s}$ and its thickness stays constant up to $150 \mathrm{~s}$, then once again thickness increases at a constant rate till it reaches $35 \mathrm{~nm}$. It has been suggested that this behaviour is due to densification of polymer film, suggesting that first there is monolayer formation to thin fibrous layer which is followed by a thick granular layer. EQCM experiments on growth of PPy suggests that initially soluble oligomer formation occurs, which upon further polymerization precipitates on anode surface. The frequency response during electropolymerization suggests second-order dependency of polymerization on monomer concentration, indicating bimolecular coupling of oxidatively formed radical cation is the rate-determining step.

During polyaniline polymerization in presence of $1 \mathrm{M}$ aromatic sulphonic acid of a constant ionic strength there is continuous rise in current after an initial decay, suggesting parallel nucleation along with autocatalytic growth of polyaniline. This aspect can be explained by analysing cyclic voltammogram (CV) during the growth of polymer by potentiodynamic method (figure 2). It can be seen from $\mathrm{CV}$ that relatively broad peak appears at potential significantly less positive than required for bulk oxidation of monomer, sharp peaks resulting from nucleation and monolayer spreading follow this. The sharp rising of these peaks suggest the formation of two dimensional patch and expanding in a shape preserving way. The close similarity of peaks in repeated cycles with increasing current suggests that nucleation occurred repeatedly on same site resulting in a nucleus on top 
of the still growing monolayer. The initial decay and then increase in current suggests that polymer formation is by random nucleation, however, we were able to observe the step type of transients in both polypyrrole and polyaniline electropolymerization by potential step technique in presence of highly functionalized dopants suggesting the formation of a layered structure. Similar type of behaviour has been reported ( $\mathrm{Li}$ and Albery 1992) for polymerization of thiophene-3-acetic acid.

\section{Conclusion}

Intercalated polyaniline and polypyrrole exhibit variety of properties depending upon stoichiometries and functional groups associated with dopant owing to their electrostatic interactions and vander Waals interactions by either close contact/overlap or both. More data is required to understand electrochemistry and charge transport, because overall redox process depends not only on monomer concentration but also on the nature of the counter ion.

\section{Acknowledgement}

The Department of Science and Technology (DST), New Delhi is acknowledged for funding a project on Organic Molecular Magnets.

\section{References}

Angeli A 1916 Gazz. Chim. Ital. 46279

Bajer I K, Pretulla J and Pron A 1994 J. Chem. Soc. Chem. Commun. 641

Baughman R H 1996 Synth. Metals 78339

Cao Y, Smith P and Heeger A J 1992 Synth. Metals 4891

Dall'Olio A, Dascola Y, Varacca V and Bocchi V 1968 Comptes

Rendus. C267 433

Desilvestro J and Scheifele W 1993 J. Mater. Chem. 3263

Dhawan S K and Trivedi D C 1991 Polym. Int. 2555

Dhawan S K and Trivedi D C 1992 J. Appl. Electrochem. 22 563

Dhawan S K and Trivedi D C $1995 J$. Appl. Polym. Sci. 58 815

Dhawan S K and Trivedi D C 1993a Bull. Mater. Sci. 16371

Dhawan S K and Trivedi D C 1993b Synth. Met. 60 63, 67

Dhawan S K, Ram M K, Chandra S and Trivedi D C 1997 Sensors and Acuators (Chemicals) B40 99

Diaz A F, Kanazawa K K, Gardini G P 1979 J. Chem. Soc. Chem. Commun. 635
Duic L J, Mandic Z and Kovacicek F 1994 J. Polym. Sci. Polyn. Chem. A32 105

Haung W S, MacDiarmid A G and Epstein A J 1987 J. Chem. Soc. Chem. Commun. 1784

Heeger A J 1995 TRIP 339

Javadi H H S, Angelopolous M, Mac Diarmid A G and Epstein A J 1988 Synth Metals 2601

Kim Y T, Collins R W, Vedam K and Allara D L $1991 \mathrm{~J}$. Electrochem. Soc. 1383266

Kumar G, Sivashanmugam A, Muniyandi N, Dhawan S K and Trivedi D C 1996 Synth. Metals 81279

Li F and Albery W J 1992 Electrochim. Acta. 37393

Litzemann, Scheerer B, Fink J, Meerholtz K, Heinze J, Saricifici N S and Kuzmany H 1989 Synth. Metals 29 E313

Letheby H $1862 \mathrm{~J}$. Am. Chem. Soc. 15161

MacDiarmid A G, Chiang J C, Halporn M, Huang W S, Mu S L, Somasiri N L, Wu W and Yaniger S I 1985 Mol. Cryst. Liq. Cryst. 121173

Mcneill R, Siudak R, Wardlaw J H and Weiss D E 1963 Aust. J. Chem. 161056

Raghunathan A, Natarajan T S, Rangarajan G, Dhawan S K and Trivedi D C 1993 Phys Rev B47 1318

Raghunathan A, Rangarajan G and Trivedi D C 1996 Synth. Metals $\mathbf{8 1} 39$

Sathyanarayanan S, Dhawan S K, Balakrishnan K and Trivedi D C 1992 Corrosion Sci. 331831

Sathyanarayanan S, Dhawan S K, Balakrishnan K and Trivedi D C 1994 Electrochimica. Acta. 39831

Schackelette L W, Wolf J F, Gould S and Baughman R H 1988 J. Chem. Phys. 883955

Shanon K and Fernandez J E $1994 \mathrm{~J}$. Chem. Soc. Chem. Commun. 643

Shim Y B and Park S M 1989 Synth. Metals 29 E169

Tang H, Kitani A and Shiotani 1996 Electrochim. Acta 411561

Trivedi D C 1994 Indian J. Chem. A33 552

Trivedi D C 1997 Hand book of organic conductive molecules and polymers: Conductive molecules and electric properties (ed.) H S Nalwa (Chichester, UK: John Wiley and Sons Ltd) vol. 2 pp 505-572

Trivedi D C and Srinivasan S 1988 J. Chem. Soc. Chem. Commun. 410

Trivedi D C 1989 J. Chem. Soc. Chem. Commun. 544

Trivedi D C and Dhawan S K 1992 J. Mater. Chem. 21091

Trivedi D C and Dhawan S K 1993a Polym. Adv. Tech. 4335

Trivedi D C and Dhawan S K 1993b Synth. Met. 58309

Trivedi D C and Dhawan S K 1993c Synth. Met. 5967

Trivedi D C 1998 Solid State Electrochem. 285

Yue J and Epstein A J $1990 \mathrm{~J}$. Am. Chem. Soc. 1122800

Zhong C, Storck W and Doblhofer K 1990 Ber. Bunseges. Phys. Chem. 941149

Zotti G, Catterin S and Comisso N 1988 J. Electroanal. Chem. 239387 